\title{
QUALIDADE FISIOLÓgICA DE SEMENTES DE SOJA SUBMETIDAS À DESSECAÇÃO EM PRÉ- COLHEITA
}

Renan Canzi Comin ${ }^{1}$, Geri Eduardo Meneghello ${ }^{2}$, Jader Job Franco ${ }^{3}$, Alexandre Moscarelli Levien ${ }^{1}$, Kassiana $\mathrm{Kehl}^{1}$, Geovan Canzi Comin ${ }^{4}$

${ }^{1}$ Fundação Pró-Sementes. ${ }^{2}$ Universidade Federal de Pelotas - UFPel, Faculdade de Agronomia Eliseu Maciel, PPG em C\&T de Sementes. ${ }^{3}$ Engenheiro Agrônomo. ${ }^{4}$ Ecomin Agroambiental. E-mail: geriem@ufpel.edu.br

\section{RESUMO}

A utilização de sementes de soja (Glycine max L.) com alta qualidade é um dos aspectos diretamente responsáveis para a obtenção de índices satisfatórios de produtividade. A prática de dessecação é uma importante aliada dos produtores para antecipação da colheita e minimização da rápida deterioração da qualidade de sementes no campo. Neste contexto, o trabalho teve como objetivo avaliar o efeito do uso de dessecantes aplicados no estádio R7 sobre a qualidade fisiológica de sementes de soja. $O$ experimento foi conduzido em área experimental, localizada no município de Passo Fundo, Rio Grande do Sul. Foram semeadas sob delineamento experimental parcela dividida, quatro cultivares de soja (FPS Jupiter RR, FPS Paranapanema RR, BRS Tordilha RR e FPS Urano RR), manejadas segundo recomendações técnicas para a cultura. Ao atingir o estádio R7 aplicou-se diferentes manejos de dessecação (sem dessecação, paraquat, diquat e glufosinato de amônio) na sub parcela. As sementes foram colhidas com colhedora de parcelas e avaliou-se o peso de mil sementes, a germinação e envelhecimento acelerado, sendo os dois últimos, avaliados aos 30 e 180 dias após a colheita. A dessecação em pré-colheita no estádio R7 da cultura da soja não afeta negativamente a qualidade fisiológica das sementes e, de modo geral, para as cultivares FPS Júpiter RR e FPS Urano RR, possibilitou a obtenção de sementes mais vigorosas aos 30 DAC. Nas condições em que o experimento foi conduzido, os herbicidas diquat, paraquat e glufosinato de amônio podem ser utilizados para dessecação em pré-colheita nos campos de produção de sementes.

Palavras-chave: herbicida; estádio fenológico; vigor; Glycine max L.

\section{PHYSIOLOGICAL QUALITY OF SOYBEAN SEEDS SUBMITTED TO PRE-HARVEST DESICCATION}

\begin{abstract}
The use of high quality soybean seeds (Glycine max L.) is one of the most important aspects to obtain satisfactory productivity indexes. The desiccation practice is an important idea of the producers to anticipate the harvest and to minimize the rapid deterioration of seed quality in the field. In this context, the objective of this work was to evaluate the effect of the use of desiccants applied in R7 stage on the physiological quality of soybean seeds. The experiment was conducted in experimental area, located in the city of Passo Fundo, Rio Grande do Sul. Four soybean cultivars (FPS Jupiter RR, FPS Paranapanema RR, BRS Tordilha RR and FPS Urano RR) were planted under experimental design, managed according to technical recommendations for the crop. Upon reaching the R7 stage, different desiccation treatments (without desiccation, paraquat, diquat and glufosinate ammonium) were applied to the subplot. The seeds were harvested with a plot harvester and the weight of one thousand seeds, germination test and accelerated aging test were evaluated, being the last two evaluated at 30 and 180 days after harvest. The pre-harvest desiccation at the R7 stage of soybean cultivation did not negatively affect the physiological quality of the seeds and, generally way, for the cultivars FPS Jupiter RR and FPS Urano RR, it was possible to obtain more vigorous seeds at $30 \mathrm{DAC}$. Under the conditions under which the experiment was conducted, the diquat, paraquat and glufosinate ammonium herbicides can be used for pre-harvest desiccation in the seed production fields.
\end{abstract}

Keywords: herbicide; phenological stages; vigor; Glycine max L. 


\section{INTRODUÇÃO}

A soja (Glycine max L.) apresenta grande importância na produção de alimentos, sendo fonte de matéria-prima para a indústria e alimentação animal, com ampla adaptação às condições brasileiras, sendo considerada a mais importante oleaginosa cultivada no mundo (LAMEGO et al., 2013). O Brasil é o segundo maior produtor mundial, com uma área plantada de 35,1 milhões de hectares na safra 2017/18 e produção de 118,8 milhões de toneladas do grão, o que representa um incremento de produção de $4,2 \%$ em relação à safra 2016/17 (CONAB, 2018). Neste cenário de produção, o uso de sementes de qualidade é indispensável para o estabelecimento de lavouras de alto rendimento.

A qualidade de um lote de sementes é resultante do somatório dos atributos genéticos, físicos, sanitários e fisiológicos (PESKE et al., 2012). Neste último, a avaliação da germinação e do vigor é de extrema importância tanto para as empresas produtoras de sementes, que utilizam essas características na tomada de decisão para a comercialização dos lotes, como para os produtores rurais, que utilizam essas informações para escolha das áreas e ajuste da densidade de semeadura da cultura, além da relação direta que existe entre a qualidade da semente utilizada e o potencial produtivo de uma lavoura $(\mathrm{SCHUCH}$ et al., 2009).

Durante o processo de formação e maturação das sementes, a máxima qualidade é observada na maturidade fisiológica da cultura (CARVALHO; NAKAGAWA, 2000). A colheita das sementes de soja nesse período seria, tecnicamente, o momento ideal, pois o grau de deterioração é mínimo (CARVALHO; NAKAGAWA, 2000). No entanto, a colheita mecânica é inviabilizada nesse período devido ao alto teor de umidade, que varia entre 45 e $60 \%$ (MARCOS FILHO, 2005). Essa fase também representa o fim do acúmulo de massa seca e do período de enchimento das sementes, cessando a conexão vascular das sementes com a planta-mãe (DALTRO et al., 2010).

A antecipação da colheita é uma prática que visa a obtenção de sementes com alta qualidade fisiológica e sanitária, reduzindo sua exposição à condições climáticas adversas (CELLA et al., 2014). Para França-Neto et al. (2010), os estresses climáticos e nutricionais, frequentemente associados a danos causados por insetos e microrganismos, são considerados como as principais causas da deterioração da semente no campo, sendo a deterioração por umidade um dos fatores que mais afetam a qualidade da semente de soja. Assim, a prática de dessecação antecipada é uma importante aliada aos produtores para minimizar as perdas de qualidade de sementes (INOUE et al., 2003). Ainda, segundo Lacerda et al. (2001) esta prática promove a antecipação da colheita de soja em até sete dias em relação à época de colheita sem o emprego da dessecação, elevando a eficiência de trabalho das colhedoras e redução das perdas (HAMER; HAMER, 2003), facilitando dessa forma a logística do produtor de sementes com o escalonamento da colheita.

O sucesso na dessecação pré-colheita depende, dentre outros fatores, do herbicida dessecante e do estádio de aplicação na cultura (PEREIRA et al., 2015). A prática de dessecação com herbicidas acelera o processo de perda de água pelas plantas e, consequentemente, das sementes, reduzindo o período de exposição a fatores bióticos e abióticos após a maturidade fisiológica (HAMER; HAMER, 2003). É fundamental avaliar a época de aplicação dos herbicidas dessecantes, sendo recomendado para a cultura da soja o estádio R7, onde 80 a $90 \%$ das vagens devem estar mudando de coloração de verde para o amarelado (CELLA et al., 2014).

Dentre os herbicidas mais utilizados no processo de dessecação em pré-colheita, destaca-se o paraquat, diquat e o glufosinato de amônio. Segundo Oliveira Junior (2011), os herbicidas paraquat e diquat (Inibidores do Fotossistema I) e glufosinato de amônio (Inibidor da Glutamina Sintase) são relativamente não seletivos, usados para controle de toda a vegetação como dessecantes aplicados em précolheita. O glufosinato de amônio, apesar de ser também um herbicida de contato, possui mais facilidade de translocação do que o paraquat (LACERDA et al., 2005).

No entanto, pesquisas demonstram reduções na germinação e no vigor após a dessecação pré-colheita, e por outro lado, estudos avaliam positivamente essa prática, destacando ausência de danos na produtividade e na qualidade das sementes produzidas, apresentando dessa forma resultados distintos na literatura (BÜLOW; CRUZ-SILVA, 2012; CELLA, et al, 2014; DALTRO et al, 2010; PEREIRA et al., 2015;). Para Marcos Filho (2005), a aplicação merece os devidos cuidados porque sua antecipação ou o atraso podem acarretar efeitos prejudiciais à qualidade do produto, exigindo 
identificação precisa da maturidade fisiológica, tomada como base para determinação de época do tratamento.

Diante do exposto, o objetivo deste trabalho foi avaliar o efeito do uso dos dessecantes paraquat, diquat e glufosinato de amônio, aplicados em R7 sobre a qualidade fisiológica de sementes de soja.

\section{MATERIAL E MÉTODOS}

O experimento foi conduzido em área experimental, localizada no município de Passo Fundo, Rio Grande do Sul, latitude: $28^{\circ} 13^{\prime} 26,6^{\prime \prime}$ e longitude: $52^{\circ} 20^{\prime} 45,7^{\prime \prime}$. O solo do local é do tipo Latossolo Vermelho Distrófico (STRECK et al., 2008). O experimento foi conduzido sob o delineamento de parcelas divididas, sendo a parcela formada pelo fator cultivares, com quatro níveis (FPS Júpiter RR, FPS Paranapanema RR, BRS Tordilha RR e FPS Urano RR) e nas sub parcelas os herbicidas (sem dessecação, paraquat, diquat e glufosinato de amônio).

A semeadura do experimento foi realizada utilizando-se uma semeadora de parcelas pneumática, com 5 linhas de plantio, acoplada a um trator New Holland TL de $75 \mathrm{CV}$. Cada parcela foi composta por 5 linhas de $4 \mathrm{~m}$ com espaçamento de $0,45 \mathrm{~m}$ entre as linhas. A adubação utilizada no plantio foi de $200 \mathrm{~kg} \mathrm{ha}^{-1}$ da fórmula (NPK) $2-18-18$ seguindo recomendação via interpretação de análise de solo. Após a emergência das plântulas, realizouse o desbaste visando padronizar o estande de plantas de acordo com as recomendações técnicas (FUNDAÇÃO PRÓ-SEMENTES, 2013).

O manejo da cultura nas parcelas experimentais foi efetuado seguindo recomendações técnicas (EMBRAPA, 2012). Foram realizadas visitas periódicas para acompanhar o desenvolvimento da cultura até atingir a fase de R7, período em que a planta apresenta uma vagem normal no caule com coloração madura, de acordo com a escala de Fehr e Caviness (1977). Nesse estádio foi realizada a aplicação dos herbicidas dessecantes, nas doses recomendadas pelos fabricantes, conforme segue: glufosinato de amônio $\left(2 \mathrm{~L} \mathrm{ha}{ }^{-1}\right)$, paraquat $\left(2 \mathrm{Lha}^{-1}\right)$ e diquat $\left(2 \mathrm{~L} \mathrm{ha} \mathrm{a}^{-1}\right)$. Em todas as caldas adicionaram-se de óleo mineral (Nimbus), a $0,2 \% \mathrm{v} / \mathrm{v}$. Os produtos foram aplicados com o auxílio de um pulverizador costal elétrico, equipado com barra com bicos tipo leque, calibrado para proporcionar um volume de aplicação de $100 \mathrm{~L} \mathrm{ha}^{-1}$.
Para a colheita foi utilizada uma colheitadeira de experimentos da marca Semina, sendo considerada como área útil apenas as três linhas centrais, excluindo-se $0,5 \mathrm{~m}$ de cada extremidade. As amostras colhidas foram acondicionadas em sacos de pano e logo em seguida levadas ao armazém da empresa Fundação Pró-Sementes, para determinação da umidade. Posteriormente, foram enviadas ao laboratório para realização dos testes de germinação, vigor e determinação do peso de mil sementes (PMS). Os testes de germinação e vigor foram realizados em duas épocas diferentes, aos 30 e 180 dias após a colheita (DAC), no laboratório de análise de sementes CebtecAGRO, visando identificar possíveis danos imediatos e latentes da utilização de dessecantes em précolheita.

O peso de mil sementes - PMS foi realizado conforme as Regras para Análise de Sementes - RAS (BRASIL, 2009). O teste foi montado utilizando-se 8 repetições de 100 sementes, as quais foram pesadas em balança de precisão, o resultado da determinação foi calculado multiplicando-se por 10 , o peso médio obtido das 8 repetições de 100 sementes.

0 teste germinação em areia foi realizado conforme a Regras para Análise de Sementes RAS (BRASIL, 2009), com 8 repetições de 50 sementes, tendo como substrato areia esterilizada, umedecidas com água destilada até atingir $60 \%$ da capacidade de campo, mantidas em sala de germinação com temperatura constante de $25^{\circ} \mathrm{C}$. No $8^{\circ}$ dia após a semeadura efetuou-se a contagem de plântulas normais, anormais e sementes mortas (BRASIL, 2009).

$O$ teste de envelhecimento acelerado foi realizado em caixas do tipo Gerbox, com uma tela de alumínio suspensa, com uma camada de sementes dispostas uniformemente, contendo no fundo da caixa $40 \mathrm{~mL}$ de água destilada. Após a montagem, as sementes foram acondicionadas em estufa Biochemical Oxigen Demand - B.O.D. a $42^{\circ} \mathrm{C}$ por um período de 48 horas. Após esse período, foram semeadas 8 repetições de 50 sementes em papel Germitest, umedecidos com o volume de água equivalente a 2,5 vezes o peso do papel. Posteriormente, as amostras foram acondicionadas no germinador a $25{ }^{\circ} \mathrm{C}$ por um período de 5 dias, contabilizado após esse período o número de plântulas normais, anormais e mortas.

Os dados obtidos foram avaliados quanto a normalidade e posteriormente submetidos à 
análise de variância. Constada significância, as médias foram comparadas entre si pelo teste de Tukey a $5 \%$ de probabilidade de erro, utilizandose o software ASSISTAT (SILVA; AZEVEDO, 2009), levando em conta os efeitos principais, ou desdobrados, de acordo com a necessidade.

\section{RESULTADOS E DICUSSÃO}

Os dados referentes à germinação das sementes de soja das quatro cultivares avaliadas, submetidas aos distintos manejos de dessecação na fase de pré-colheita, estão apresentados na Tabela 1.

Não houve significância estatística para a variável germinação aos 30 após a colheita - DAC. Considerados os valores absolutos, todas as cultivares, independentemente do manejo empregado, apresentaram germinação mínima de $97 \%$. Esses resultados permitem inferir que os herbicidas nas doses e formulações utilizados, aplicados no estádio R7, não afetaram negativamente a germinação das sementes. Os resultados obtidos corroboram com Daltro et. al.
(2010), que observaram que o uso dos dessecantes paraquat, diquat, paraquat+diquat e paraquat+diuron não afetaram o rendimento e a qualidade fisiológica de sementes de soja, independente do estádio de aplicação em R6.5 ou R7. Estudo também demonstrou que a dessecação química com o uso de glufosinato de amônio e paraquat, aplicados no estádio R7.1, possibilitou antecipar a colheita em seis dias, mantendo o percentual de germinação, sem influenciar negativamente a produtividade de sementes (PEREIRA et al., 2015). No entanto, Bülow e Cruz-Silva (2012) observaram que a aplicação de paraquat ou glyphosate em précolheita da soja, afetou negativamente os parâmetros fisiológicos de germinação e vigor de sementes, não sendo recomendado o uso desses produtos em pré-colheita da cultura da soja. Aos 180 DAC não foram observadas diferenças significativas para a variável germinação entre as combinações de tratamentos avaliados.

Tabela 1. Germinação de sementes de soja aos 30 e 180 dias após a colheita (DAC), com quatro cultivares manejadas em pré-colheita com herbicidas dessecantes. Passo Fundo-RS, 2014.

\begin{tabular}{|c|c|c|c|c|c|}
\hline \multirow[b]{2}{*}{ Cultivar } & \multicolumn{4}{|c|}{ Germinação 30 DAC (\%) } & \multirow[b]{2}{*}{ Media } \\
\hline & Diquat $^{\text {ns }}$ & $\begin{array}{c}\text { Gluf. } \\
\text { Amônio }^{\text {ns }}\end{array}$ & Paraquat $^{\text {ns }}$ & $\begin{array}{c}\text { Sem } \\
\text { Dessec. }^{\text {ns }}\end{array}$ & \\
\hline FPS Jupiter RR ${ }^{\text {ns }}$ & 99 & 97 & 98 & 98 & 98 \\
\hline FPS Paranapanema RR ${ }^{\text {ns }}$ & 97 & 97 & 97 & 99 & 98 \\
\hline BRS Tordilha RR ${ }^{\text {ns }}$ & 98 & 98 & 98 & 98 & 98 \\
\hline FPS Urano RR $R^{\text {ns }}$ & 98 & 97 & 98 & 97 & 98 \\
\hline Media & 98 & 97 & 98 & 98 & \\
\hline \multicolumn{6}{|l|}{ C.V.a $1,10 \%$} \\
\hline \multicolumn{6}{|l|}{ C.V.b 1,30\% } \\
\hline \multirow[b]{2}{*}{ Cultivar } & \multicolumn{4}{|c|}{ Germinação 180 DAC (\%) } & \multirow[b]{2}{*}{ Media } \\
\hline & Diquat $^{\text {ns }}$ & $\begin{array}{c}\text { Gluf. } \\
\text { Amônio }^{\text {ns }}\end{array}$ & Paraquat $^{\text {ns }}$ & $\begin{array}{c}\text { Sem } \\
\text { Dessec. }\end{array}$ & \\
\hline FPS Júpiter RR ${ }^{\text {ns }}$ & 92 & 91 & 88 & 89 & 90 \\
\hline FPS Paranapanema RR ${ }^{\text {ns }}$ & 85 & 88 & 89 & 82 & 86 \\
\hline BRS Tordilha RR ${ }^{\mathrm{ns}}$ & 89 & 89 & 89 & 85 & 88 \\
\hline FPS Urano RR ${ }^{\text {ns }}$ & 84 & 91 & 81 & 87 & 86 \\
\hline Media & 88 & 89 & 87 & 86 & \\
\hline \multicolumn{6}{|l|}{ C.V.a $8,50 \%$} \\
\hline C.V.b 6,80\% & & & & & \\
\hline
\end{tabular}


Diante disso, nas condições em que a pesquisa foi realizada, a prática de dessecação proporcionou uma resposta positiva na qualidade de sementes, garantindo uma germinação acima do mínimo exigido de $80 \%$ para comercialização (BRASIL, 2013), aos 30 e 180 DAC. Dessa forma, infere-se que não houve prejuízos por danos latentes, tornando a dessecação em pré-colheita uma técnica promissora para o produtor de sementes. Para Lacerda et al. (2003), o potencial de conservação de sementes de soja depende diretamente da qualidade fisiológica das mesmas no início do período de armazenamento, sendo intimamente relacionada ao momento da colheita.

Para a variável envelhecimento acelerado (E.A.), verificou-se interação significativa entre a aplicação dos dessecantes e as cultivares de soja (Tabela 2).

Aos 30 DAC, considerando-se os tratamentos com herbicidas, não foi verificada redução no vigor das sementes para a aplicação dos dessecantes diquat e glufosinato de amônio, independente da cultivar avaliada. Já para o herbicida paraquat, observou-se redução no vigor para as cultivares FPS Paranapanema RR e FPS Urano RR no mesmo período. Esse resultado difere do encontrado por Guimarães et al. (2012), que observaram que $\mathrm{o}$ herbicida paraquat promoveu os melhores índices de germinação e vigor de sementes de soja quando utilizado nos estádios R6 e R7.2. Conforme descrito por Cella et al., (2014) as respostas com relação às perdas causadas pela dessecação antecipada, em parte, dependem da cultivar em estudo.

Não foram observadas reduções de vigor para as cultivares avaliadas, comparando-se o uso dos herbicidas em pré-colheita com o tratamento sem dessecação, aos 30 DAC. Ressalta-se que para as cultivares FPS Júpiter RR e FPS Urano RR aos 30 DAC, a prática de dessecação em précolheita possibilitou a obtenção de sementes mais vigoras em comparação ao tratamento sem dessecação em pré-colheita, exceto para o uso do herbicida paraquat na cultivar FPS Urano RR. Guimarães et al. (2012) verificaram que a prática de dessecação em pré-colheita com os herbicidas paraquat (estádios R6 e R7.2) e glufosinato de amônio (estádio R7.2), também resultaram em sementes mais vigoras em comparação ao tratamento sem dessecação. Por outro lado, Lamego et al. (2013) verificaram redução no vigor de sementes de soja quando a cultivar Energia RR foi dessecada com paraquat no estádio R7.1, apresentando-se mais vigorosas as sementes provenientes do tratamento testemunha.

Analisando também o envelhecimento acelerado em sementes de soja após a aplicação dos herbicidas paraquat e diquat nos estádios R6.0, R7.1 e R7.2 e R7.3, Kappes et al. (2009) observaram que as sementes oriundas de plantas que não foram submetidas a pré dessecação originaram os maiores percentuais de plântulas normais, diferindo das plantas dessecadas nos três primeiros estágios, sendo justificado pela imaturidade fisiológica das sementes por ocasião das aplicações. No mesmo estudo, na dessecação efetuada no estádio R7.3, os maiores percentuais de germinação após o teste de E.A. foram obtidos para os tratamentos com $\mathrm{o}$ paraquat $\mathrm{e} a$ testemunha. 
Tabela 2. Teste de envelhecimento acelerado de sementes de soja, aos 30 e 180 dias após a colheita, com quatro cultivares manejadas em pré-colheita com herbicidas dessecantes. Passo Fundo-RS, 2014.

\begin{tabular}{|c|c|c|c|c|c|}
\hline \multirow[b]{2}{*}{ Cultivar } & \multicolumn{4}{|c|}{ Envelhecimento Acelerado (\%) - 30 Dias } & \multirow[b]{2}{*}{ Média } \\
\hline & Diquat & $\begin{array}{c}\text { Gluf. } \\
\text { Amônio }\end{array}$ & Paraquat & Sem Dessec. & \\
\hline FPS Júpiter RR & 99 aA & $94 \mathrm{aA}$ & 95 aA & $78 \mathrm{bB}$ & $92 b$ \\
\hline FPS Paranapanema RR & $90 \mathrm{aA}$ & $93 \mathrm{aA}$ & $88 \mathrm{bA}$ & $95 \mathrm{aA}$ & $91 \mathrm{~b}$ \\
\hline BRS Tordilha RR & 97 aA & 95 aA & 97 aA & 97 aA & $96 a$ \\
\hline FPS Urano RR & $91 \mathrm{aA}$ & 91 aA & $79 \mathrm{bB}$ & $84 \mathrm{bB}$ & $86 \mathrm{c}$ \\
\hline Media & $94 \mathrm{~A}$ & $93 \mathrm{~A}$ & $90 \mathrm{~B}$ & $89 \mathrm{~B}$ & \\
\hline \multicolumn{6}{|l|}{ C.V.a $6,30 \%$} \\
\hline \multicolumn{6}{|l|}{ C.V.b $6,70 \%$} \\
\hline \multirow[b]{2}{*}{ Cultivar } & \multicolumn{4}{|c|}{ Envelhecimento Acelerado (\%) - 180 Dias } & \multirow[b]{2}{*}{ Média } \\
\hline & Diquat & Gluf. Amônio & Paraquat & $\begin{array}{c}\text { Sem } \\
\text { Dessec. }\end{array}$ & \\
\hline FPS Júpiter RR & 85 aA & $84 \mathrm{aA}$ & $84 \mathrm{aA}$ & $74 \mathrm{bA}$ & $81 \mathrm{a}$ \\
\hline FPS Paranapanema RR & $62 \mathrm{aA}$ & $72 \mathrm{aA}$ & $58 \mathrm{bA}$ & $60 \mathrm{bA}$ & $63 \mathrm{~b}$ \\
\hline BRS Tordilha RR & 79 aA & $93 \mathrm{aA}$ & 88 aA & $92 \mathrm{aA}$ & $88 a$ \\
\hline FPS Urano RR & 73 aA & $81 \mathrm{aA}$ & $70 \mathrm{bA}$ & 79 aA & $75 a$ \\
\hline Media & $75 \mathrm{~A}$ & $82 \mathrm{~A}$ & $75 \mathrm{~A}$ & $76 \mathrm{~A}$ & \\
\hline \multicolumn{6}{|l|}{ C.V.a $15,10 \%$} \\
\hline C.V.b $15,20 \%$ & & & & & \\
\hline
\end{tabular}

Para a variável envelhecimento acelerado aos 180 DAC, as cultivares FPS Paranapanema RR e FPS Urano RR apresentaram os menores níveis de vigor quando da aplicação do herbicida paraquat. Para os demais tratamentos com dessecantes, não foram observadas diferença significativas, independente da cultivar. No tratamento sem dessecação, as cultivares FPS Júpiter RR e FPS Paranapanema RR, apresentaram vigor de $74 \%$ e $60 \%$, respectivamente, diferindo negativamente das demais cultivares avaliadas. Nota-se ainda, que os valores médios de germinação aos 180 DAC após o teste de E.A. revelaram redução significativa no vigor para a cultivar FPS Paranapanema RR.

Analisando-se o efeito dos herbicidas, não foram verificadas reduções significativas de vigor após 180 DAC, comparativamente ao tratamento testemunha. Dessa forma, os resultados obtidos para a cultivar FPS
Panapanema RR podem estar associadas a características genéticas intrínsecas da cultivar.

Para a variável peso de mil sementes (PMS), não foram observadas diferenças significativas entre o tratamento sem dessecação e os tratamentos com dessecantes, independente da cultivar (Tabela 3). Em relação aos diferentes herbicidas dessecantes aplicados, as cultivares BRS Tordilha RR e FPS Urano RR, de modo geral, apresentaram os maiores valores de PMS em comparação as outras cultivares. $O$ peso de mil sementes é uma importante variável para analisar os diversos estresses a que uma planta pode enfrentar, dentre eles aqueles decorrentes da aplicação de produtos nas lavouras de soja, na qual as plantas podem responder de maneira positiva ou negativa. 
Tabela 3. Peso de mil sementes (PMS) de quatro cultivares de soja em função do manejo pré-colheita com dessecantes químicos. Passo Fundo-RS, 2014.

\begin{tabular}{|c|c|c|c|c|c|}
\hline \multirow{2}{*}{ Cultivar\Produto } & \multicolumn{4}{|c|}{ PMS (g) } & \multirow{2}{*}{ Media } \\
\hline & Diquat & Gluf. Amônio & Paraquat & Sem Dessec. & \\
\hline FPS Júpiter RR & $148,7 \mathrm{bA}$ & $150,5 \mathrm{bA}$ & $150,0 \mathrm{bA}$ & $149,8 \mathrm{aA}$ & $149,8 b$ \\
\hline FPS Paranapanema RR & $153,9 \mathrm{bA}$ & $151,0 \mathrm{bA}$ & $147,0 \mathrm{bA}$ & 155,4 aA & $151,8 b$ \\
\hline BRS Tordilha RR & 163,3 aA & $163,8 \mathrm{aA}$ & 163,9 aA & $160,5 \mathrm{aA}$ & $162,9 \mathrm{a}$ \\
\hline FPS Urano RR & 158,0 aA & $150,5 \mathrm{bA}$ & 162,4 aA & 158,4 aA & $157,3 \mathrm{a}$ \\
\hline Media & $155,9 \mathrm{~A}$ & $153,9 \mathrm{~A}$ & $155,8 \mathrm{~A}$ & $156,0 \mathrm{~A}$ & \\
\hline
\end{tabular}

C.V.a $5,80 \%$

C.V.b $4,10 \%$

Médias seguidas de mesma letra maiúsculas na linha, e minúsculas na coluna, não diferem entre si pelo teste de Tukey a $5 \%$ de probabilidade de erro.

Diferentes desempenhos são observados na literatura em relação ao PMS após a aplicação de dessecantes nas plantas. Silva et al. (1999) não observaram diferenças significativas para a massa de mil sementes e diferentes dessecantes aplicados na cultura do feijão. Entretanto, Pelúzio et al. (2008) detectaram quedas significativas no peso de 100 sementes para dessecação com paraquat nos estádios R6 e R7, em comparação a testemunha. Lamego et al., (2013) também verificaram redução no PMS para a dessecação com paraquat nos estádios R6 e R7.1.

A colheita antecipada proporciona a obtenção de sementes de soja com melhor qualidade fisiológica (DALTRO et al., 2010), tendo-se em vista que o retardamento da colheita após a maturação fisiológica constitui uma das principais causas da redução da germinação e vigor (SEDYAMA, 2013). No entanto, Lacerda et al. (2005) afirmam que é necessário considerar alguns aspectos importantes quando se pretende usar dessecantes químicos, como o modo de ação do produto, as condições ambientais em que esse é aplicado, o estádio fenológico em que a cultura se encontra, a eventual ocorrência de resíduos tóxicos no material colhido, a influência na produção, germinação e vigor de sementes.

\section{CONCLUSÕES}

A dessecação em pré-colheita no estádio R7 da cultura da soja não afeta negativamente a qualidade fisiológica das sementes. Para as cultivares FPS Júpiter RR e FPS Urano RR, a prática de dessecação em pré-colheita, de modo geral, possibilitou a obtenção de sementes mais vigorosas aos 30 DAC.
Não foram observadas diferenças significativas no PMS entre o tratamento sem dessecação e os tratamentos com dessecantes, independente da cultivar.

Nas condições em que o experimento foi conduzido e para as cultivares avaliadas, os herbicidas diquat, paraquat e glufosinato de amônio podem ser utilizados para dessecação em pré-colheita nos campos de produção de sementes.

\section{REFERÊNCIAS BIBLIOGRÁFICAS}

BRASIL. Ministério da Agricultura, Pecuária e Abastecimento. Instrução Normativa $\mathrm{n}^{\circ} \mathbf{4 5}$ de $\mathbf{1 7}$ de dezembro de 2013. Brasília: Mapa, 2013. 38p.

BRASIL. Ministério da Agricultura, Pecuária e Abastecimento. Regras para análise de sementes. Brasília: Mapa, 2009. 399 p.

BÜLOW, R.L.; CRUZ-SILVA, C.T.A. Dessecantes aplicados na pré-colheita na qualidade fisiológica de sementes de soja. Journal of Agronomic Sciences, v.1, n.1, p.67-75, 2012.

CARVALHO, N.M.; NAKAGAWA, J. Sementes: ciência, tecnologia e produção. 4. ed. Jaboticabal: FUNEP, 2000. 588p.

CELLA, V.; SILVA, J.F.; AZEVEDO, P.H.; AZEVEDO, V.H.; HOFFMAN, L.L. Efeito da dessecação em estádios fenológicos antecipados na cultura da soja. Bioscience Journal, v.30, n.5, p.1364-1370, 2014.

CONAB. Acompanhamento da Safra Brasileira Grãos, Safra 2017/18 - n.10. Disponível em: 
https://www.conab.gov.br/info-agro/safras.

Acesso em: 30 jul. 2018.

DALTRO, E.M.F.; ALBUQUERQUE, M.C.F.; FRANÇA-NETO, J.B.; GUIMARÃES, S.C.; GAZZIERO, D.L.P.; HENNING, A.A. Aplicação de dessecantes em pré-colheita: efeito na qualidade fisiológica de sementes de soja. Revista Brasileira de Sementes, v.32, n.1, p.111-122, 2010. http://dx.doi.org/10.1590/S0101-

$\underline{31222010000100013}$

EMBRAPA. Indicações técnicas para a cultura da soja no Rio Grande do Sul e em Santa Catarina, safras 2012/2013 e 2013/2014. 2012. Disponível em:

https://www.agrolink.com.br/downloads/Indica \%C3\%A7\%C3\%B5es\%20T\%C3\%A9cnicas\%20para \%20a\%20cultura\%20da\%20soja\%20no\%20RS\%20 e\%20SC\%202013-2014.pdf Acesso em: 16 jan. 2019.

FEHR, W.R.; CAVINESS, C.E. Stages of soybean development. Agricultural Experimental Station Special Report, lowa, v.80, p.1-11, 1977.

FRANÇA-NETO, J.B.; KRZYZANOWSKI, F.C.; HENNING, A.A.; PÁDUA, G.P. Tecnologia da produção de semente de soja de alta qualidade. Informativo Abrates, v.20, n.3, p.26-32, 2010.

FUNDAÇÃO PRÓ-SEMENTES. Cultivares de soja FPS. $2013 . \quad$ Disponível em: http://www.fundacaoprosementes.com.br/cultiv ares/soja. Acesso em 15 jan. 2019.

GUIMARÃES, V.F.; HOLLMANN, M.J.; FIOREZE, S.L.; ECHER, M.M.; ODRIGUES-COSTA, A.C.P.; ANDREOTTI, M. Produtividade e qualidade de sementes de soja em função de Estádios de dessecação e herbicidas. Planta Daninha, v.30, n.3, p.567-573, 2012. http://dx.doi.org/10.1590/S010083582012000300012

HAMER, E.; HAMER, E. Produção de sementes requer planejamento. Seed News, n.4, p.23-27, 2003.

INOUE, M.H.; MARCHIORI JR., O.; BRACCINI, A.L.; OLIVEIRA JR., R.S.; ÁVILA, M.R.; CONSTANTIN, J. Rendimento de grãos e qualidade de sementes de soja após a aplicação de herbicidas dessecantes. Ciência Rural, v.33, n.4, p.769-770,
2003. http://dx.doi.org/10.1590/S0103$\underline{84782003000400030}$

KAPPES, C.; CARVALHO, M. A. C.; YAMASHITA, O. $M$. Potencial fisiológico de sementes de soja dessecadas com diquat e paraquat. Scientia Agraria., v.10, n.1, p.1-6, 2009. http://dx.doi.org/10.5380/rsa.v10i1.12520

LACERDA, A.L.S.; LAZARINI, E.; SÁ, M.E.; VALÉRIO FILHO, W.V. Aplicação de dessecantes na cultura de soja: antecipação da colheita e produção de sementes. Planta Daninha, v.19, n.3, p.381-390, $2001 . \quad$ http://dx.doi.org/10.1590/S010083582001000300011

LACERDA, A.L.S.; LAZARINI, E.; SÁ, M.E.; VALÉRIO FILHO, W.V. Armazenamento de sementes de soja dessecadas e avaliação da qualidade fisiológica, bioquímica e sanitária. Revista Brasileira de Sementes, v.25, n.2, p.97-105, 2003. http://dx.doi.org/10.1590/S0101-

$\underline{31222003000400014}$

LACERDA, A. L. S.; LAZARINI, E.; SÁ, M.E.; VALÉRIO FILHO, W.V. Efeitos da dessecação de plantas de soja no potencial fisiológico e sanitário das sementes. Tecnologia de sementes. Bragantia, v.64, n.3, p.447-457, 2005. http://dx.doi.org/10.1590/S000687052005000300015

LAMEGO, F.P.; GALLON, M.; BASSO, C.J.; KULCZYNSKI, S.M., RUCHEL, Q., KASPARY, T.E.; SANTI, A.L. Dessecação pré-colheita e efeitos sobre a produtividade e qualidade fisiológica de sementes de soja. Planta Daninha, v.31, n.4, p.929-938, 2013. http://dx.doi.org/10.1590/S010083582013000400019

MARCOS FILHO, J. Fisiologia de sementes de plantas cultivadas. Piracicaba: FEALQ, 2005. 495p.

OLIVEIRA JUNIOR, R.S. Introdução ao controle químico. In: OLIVEIRA JUNIOR, R.S.; CONSTANTIN, J.; INOUE, M.H. Biologia e Manejo de Plantas Daninhas. Curitiba: Omnipax, 2011. p.125-139.

PELÚZIO, J. M.; RAMO, L.M.; FIDELIS, R.R.; AFFÉRRI, F.S.; CASTRO NETO, M.D.; CORREIA, M.A.R. Influência da dessecação química e retardamento de colheita na qualidade fisiológica 
de sementes de soja no sul do Estado do Tocantins. Bioscience Journal, v.24, n.2, p.77-82, 2008.

PEREIRA, T.; COELHO, C.M.M.; SOUZA, C.A.; MANTOVANI, A.; MATHIAS, V. Dessecação química para antecipação de colheita em cultivares de soja. Semina: Ciências Agrárias, v.36, n.4, p.2383-2394, 2015.

PESKE, S.T.; BARROS, A.C.S.A.; SCHUCH, L.O.B. Produção de sementes. In: PESKE, S.T.; VILLELA, F.A.; MENEGHELLO, G.E. Sementes: fundamentos científicos e tecnológicos. 3. ed. Pelotas: Ed. Universitária, 2012. p.13-100.

SCHUCH, L.O.B.; KOLCHINSKI E.M.; FINATTO, J.A. Qualidade fisiológica da semente e desempenho de plantas isoladas em soja. Revista Brasileira de Sementes, v.31, n.1, p.144-149, 2009. http://dx.doi.org/10.1590/S0101-

$\underline{31222009000100016}$

SEDYAMA, T. Tecnologia e produção de sementes de soja. Londrina: Mecenas, 2013. $352 p$.

SILVA, A.A.; DOMINGOS, M.; CARDOSO, A.A. Efeitos do paraquat e da mistura paraquat + diquat, como dessecantes, aplicados em diferentes épocas, no rendimento e na qualidade fisiológica das sementes de feijão. Revista Ceres, v.46, p.239-250, 1999.

SILVA, F.A.S.E.; AZEVEDO, C.A.V. Principal Components Analysis in the Software AssistatStatistical Attendance. In: WORLD CONGRESS ON COMPUTERS IN AGRICULTURE, 7. Proceedings [...] USA: American Society of Agricultural and Biological Engineers, 2009.

STRECK, E.V.; KÄMPF, N.; DALMOLIN, R.S.D.; KLAMT, E.; NASCIMENTO, P.C.; SCHNEIDER, P.; GIASSON, E.; PINTO, L.F.S. Solos do Rio Grande do Sul. 2. ed. Porto Alegre: EMATER/RS 2008. 222p.

Recebido para publicação em 14/10/2018

Revisado em 19/01/2019

Aceito em 19/01/2019 